\title{
Online Social Transparency in Enterprise Information Systems: Risks and Risk Factors
}

\author{
Tahani Alsaedi, Keith Phalp, Raian Ali \\ Faculty of Science and Technology, Bournemouth University, UK \\ \{talsaedi, kphalp, rali\}@bournemouth.ac.uk
}

\begin{abstract}
Enterprises integrate social networking within their information systems to enhance collegiality, situational awareness, coordination and collaboration amongst their members. Social networking features can be seen in traditional systems such as the online profile, calendar, dashboard, auto-reply and status. More specialised systems enable bespoke features to declare and share and retrieve current and past engagements, team memberships, allocated tasks and priorities. Such social transparency is typically voluntary and not strictly enshrined by organisational governance and norms. Despite its positive connotations, negative consequences such as information overload, social loafing and undesired pressure can be a result of it. We conducted a multistage qualitative study, including focus groups, interviews and observations, to conceptualise online social transparency and explore the risks that stem from its unmanaged implementation. Our research aims to provide the first step towards a systematic method for risk identification and mitigation around online social transparency.
\end{abstract}

Keywords: Social transparency, Enterprise social software, Enterprise information systems.

\section{Introduction}

In enterprise information systems, it is becoming common for employees to share more information about themselves so that they enhance situational and context awareness and, hence, communication relevance and sensitivity. Enterprise social software (ESS) is an online platform that allows employees to communicate, in realtime, information about their identities, activity streams, assigned tasks, work progress and collaboration with others [1] and it allows companies to improve the business relationships with customers [2]. These platforms used to practice social transparency that we defined in our previous work as "voluntarily use of online platforms by employees to share their own information about their situation, roles and responsibilities with other members of the organisation" [3].

At the organisational level, [4] stated that transparency is one of the trends in corporate social responsibilities (CSR) that results in an improvement in enterprise performance, productivity and profit. On the individual level, social transparency is typically to enhance situational awareness, coordination, and collaboration amongst employees. Organisational scientists have long understood that the success of organisations depends on commitments, coordination, and collaboration of their employees [5]. Online social transparency is one of the mechanisms whose effects on employee collaboration and motivation have been proven [6]. Social transparency contributes to 
building trust and bringing awareness amongst organisation members through intentional information disclosure, group knowledge sharing or individual activity sharing [7]. Thus, social transparency has become a popular requirement for employees before engaging in the activities of others.

Despite the positive connotations, it seems that current digital tools are both primitive and cumbersome. Most of the enterprise social software has substantial shortcomings in regard to their facilitation of online social transparency. The ad-hoc practice of such transparency may lead to adverse effects such as disturbance, information overload and lack of interest [8]. Research on transparency and its effects is rare in the requirements engineering literature. In [9], a conceptual framework is proposed to facilitate the adoption of transparency in responsible organisational business management. It describes what organisations can do to be more transparent and the potential benefits of transparency. An argumentation framework was proposed in [10] to elicit transparency-related requirements. TranspLan language was proposed in [11], and it provides models and templates for specifying transparency requirements within enterprises and algorithms to reason about them in terms of consistency and conflicts. In terms of social transparency, the authors in [12] examined how individuals use information about others' actions in open social software and provide suggestions about the design of social media for large scale enterprise, and imply a variety of ways that transparency can support innovation, knowledge sharing and community building. In [13], it is argued that the online platforms for social transparency are making information visible without careful thoughts to the social inferences the platform design supports. The authors provided a theoretical framework for analysing social transparency and inferences stemming from the change in technology.

Although these works illuminate the potential promise of managing social transparency in the enterprise, particularly in their online platforms, scholars still handle social transparency as an information quality issue. In our work, we address the question of how to manage social transparency as an informed decision and behaviour. We provided earlier our definition of social transparency, and we assume that this transparency is an autonomous decision by organisation members to be open when conveying social information through online platforms.

In this paper, we build on our previous work in $[3,14]$ and conduct a multi-stage qualitative study (described in Section 2) to provide empirical evidence that online social transparency can lead to a negative impact on employees as well as organisations if implemented and conducted in an unmanaged style (Section 3). We provide common risks and associate them with certain classifications of online social transparency (Section 4). Our results are meant to inform future risks assessment method of online social transparency and, also, the engineering of enterprise social software so that it plays a role in guiding and steering it and make employees decisions about it more informed.

\section{Research Method}

A multistage qualitative study was used to investigate the potential shortcomings of applying social transparency within enterprise information systems. We used multiple data collection methods as presented in Table 1. The study aimed to (i) identify 
Table 1. Research Method Stages

\begin{tabular}{ccc} 
Stage & Description & Purpose \\
\hline - Review related literature including organisa- & - To conceptualise online social \\
transparency & tional transparency, CSCW, Group Dynamics, \\
Foundations & situational awareness and organisational culture & - To identify essential factors for \\
& - Two scenario-based focus groups with 14 & assessing online social transpar- \\
& participants & ency [14]
\end{tabular}

\begin{tabular}{ccc}
$\begin{array}{c}\mathbf{2}^{\text {nd }} \text { Stage } \\
\text { Exploration }\end{array}$ & Semi-structured Interview with 15 Participants & $\begin{array}{c}\text { - To build a reference model for } \\
\text { the assessment method of online } \\
\text { social transparency [3] }\end{array}$ \\
$\begin{array}{c}\text { To form an initial set of risks } \\
\text { and their factors }\end{array}$ \\
$\begin{array}{c}\mathbf{3}^{\text {rd }} \text { Stage } \\
\text { Refinement }\end{array}$ & Observation study, Interviews, Focus group & $\begin{array}{c}\text { - To form the final set of risks } \\
\text { and risk factors }\end{array}$ \\
\hline
\end{tabular}

the assessment dimensions of online social transparency and (ii) explore the typical risks and risk factors around the unmanaged conduct of it.

All the studies were recorded and transcribed verbatim to support the analysis stage. We used a thematic analysis approach by following the six phases of thematic analysis proposed by [15]. In the analysis stage, we identified the participants' views on their transparency expectations from their co-workers and managers and their concerns about affecting their role, social dependencies and actions. We used the findings of each stage as a template to start with when analysing the next stage and expanded it until we reached the saturation point in all stages. The study was approved by the research ethics committee of the authors' institution.

\subsection{Focus groups phase}

A total of 14 individuals participated in two focus group sessions to explore how they view online social transparency in the workplace, their requirements of it, and how certain modalities and configurations of transparency contribute to risks that affect aspects of their work environment. We recruited participants who worked in organisations where their role involved collaborative work with others online. The participants were given a presentation to familiarise themselves with the context of the research. We developed four scenarios to cover various aspects of transparency such as its content (e.g. intentions, plans and status), its presentation (media and interfaces), its timing and relevance. We used the scenarios in the session to stimulate discussions. Each scenario included questions to be answered individually before discussing it within the group.

\subsection{Interview phase}

We used the findings from the focus group stage as a foundation for further investigation. We conducted a semi-structured interview study to (i) confirm and refine the 
findings that related to the transparency assessment factors resulting from the focus group and (ii) explore families of typical risks related to unmanaged online transparency around those factors. While we used typical scenarios in the focus group study, the interviews were intended to delve into the personal experiences of the participants about online transparency in their workplaces. The interviews phase sought to explore the risk of transparency through the professionals' lived experiences and different work environments. We interviewed 15 participants throughout two stages. We first interviewed participants from diverse work environments, including academia, small companies and call centres. Ten employees agreed to participate in this study (four females and six males) aged between 27 and 43 years. In the second stage, we interviewed professionals in managerial roles. Five managers from various levels of seniority participated in this study that they are a project manager, call centre manager, team leader and two supervisors. We also considered diversity in gender in the second stage, with two females and three males aged between 36 to 52 years.

\subsection{Observation phase}

We conducted a two days observation study at two multicultural small-scale companies to further explore the risks and their factors of social transparency in enterprise social software and confirm they exist in real context. The companies use software called Slack (https://slack.com/intl/en-gb/enterprise) for tracking the progress, managing employees' collaboration and improving overall performance. A short interview with employees was conducted after the observation of their behaviour and interaction with the software finished.

After two days of observation, we conducted one focus group in each company. To enrich the results with a diversity of opinions originating from various perspectives, participants with managerial roles and employees were involved. As a result, a total of 10 professionals from the two companies participated in our focus group study, one session with 5 professionals in each company. In addition to the diversity in roles, we considered diversity in gender and age, with three females and seven males ranging from 28 to 49 years old. The focus group included two activities. The first was a scenario-based discussion where the scenarios used are built based on the observations made in the two companies. The second involved an open card sorting activity to organise the risks of online social transparency into groups. The card sorting aimed to confirm and refine the findings with regards to the risks of online social transparency on employees' wellbeing and performance. The card sorting activity included risks generated from participants' answers and risks founded in the second stage. Each focus group lasted for around two hours. The sessions were audio-recorded and transcribed for content analysis.

\section{Categories of Online Social Transparency}

Social transparency can be classified into three categories based on two factors (i) the awareness of the information provider and (ii) the accessibility level of the information receiver. The awareness of the information provider refers to the conscious 
choice for the information to be visible. Awareness is a spectrum, and it falls between two edge cases:

- "I revealed" case: It refers to the deliberate sharing of individual information with consent to be visible to others. For example, staffs consciously reveal information about their current status and progress in the task with the purpose that this information will be visible to enterprise members and how it can be usually used.

- "I did not reveal" case: It refers to the sharing of individual information without full awareness of the sharing action itself or the audiences of the information shared. For example, a team leader shares information about their team, where members are not aware of that. Another example is about sharing location data and not being aware or able to predict whether this might be occasionally re-shared by others.

Regarding the second factor, we found that online social transparency sharing can be classified into two kinds based on the accessibility level by enterprise members.

- Open accessibility: In which information is accessible by all individuals in the workplace. Staff open online calendar is a typical example where others can see the schedule of their colleagues and what the meetings are about and their location.

- Regulated accessibility: In which information is limited, deliberately or due to connectivity and contextual barriers, to a set of individuals in the workplace. Group conversation and to-do-list is a typical example.

Based on the two dimensions of awareness and accessibility, we found four categories of online social transparency:

- Open social transparency refers to sharing information about the self with full awareness and also desire to be visible to others in the organisation. This kind of transparency is typically motivated by increasing awareness in the workplace which will then positively affect the organisational goals. There are several examples of open social transparency in organisations such as staff calendar; staff profiles page, their location and activity status and public posts and conversations.

- Regulated social transparency refers to sharing information about the self, which reaches only specific members of the organisation. This kind of transparency regulates the visibility of individual information for various reasons, including the protection from misuse and the reduction of misconceptions amongst members. For example, sharing information about personal difficulties in the work may be of interest only to the teams to which the employee belongs. Busy colleagues may not see them due to applying filters and techniques like muting other's people status. In other words, regulation of accessibility can also be a receiver choice.

- Unconscious social transparency refers to the visibility of individual information without awareness from the information owner. This kind of transparency is one of the ethical issues in the workplace as colleagues may share personal information about an individual without their knowledge. We emphasise here that social transparency has loose contractual settings and access control and relies mainly on personal judgement and organisational and cultural norms. For example, a member of 
a team may share information about difficulties and their peers' weaknesses or peer's progress in a collaborative task with other teams aiming for external support. Such transparency seems unavoidable even in an ordinary social environment but still undesired as it has a diverse impact on the collaboration between organisational members. A participant highlighted that "it happened in the joint work when people want to jeopardise the progress or displacing colleagues form their assigned tasks."

- No social transparency refers to the situation where enterprise members are not sharing information about their activities stream, progress, and interest in certain tasks or their relationships with other colleagues. We found this case more in new members who still have not built a trust relationship with peers and management and confidence in their role and contribution to the group. Introversion and extroversion can also be part of the personality.

\section{$4 \quad$ Risks Associated with Online Social Transparency}

Our results from the first and second stages of the study indicated that risks are related to the delivery of the information in four aspects: content, timeliness, presentation and intended recipients [3]. The results of our third stage confirmed our previous findings and explored other dimensions of risk factors that need to be considered in the assessment process. Our analysis grouped the risks based on their influence area into (i) performance, (ii) wellbeing and (iii) workplace environment. Two main risk factors seemed to be prominent; the level of transparency (Section 4.1) and the way it is practised (Section 4.2).

\subsection{Risks Related to Level of Transparency}

The level of transparency indicates whether it is adequate, abundant or unsatisfactory. The level is not only determined by the information content but is inherently dependent on the reachability, relevance and interpretability of information. In other words, it is a contextual and subjective measure, determined mainly by the audiences and dependent on their personal, technical and social context. Table 2 presents the main categories of risks revolving around the different levels of transparency.

Risks related to excessive online social transparency. Excessive transparency refers to the redundant and repetitive voluntary sharing of information. It refers to pushing information overly in terms of amount and frequency. We reiterate here that the judgement of the level of transparency, in terms of information and their meaningfulness, is not uniform but depends on the recipients personal, task, technical and social context. Examples of these contexts are the recipient's availability, workloads, time, preferences, location, and available communication bandwidth.

Performance. Excessive transparency can lead to isolating individuals from others. We noted that participants may avoid collaboration with colleagues who practice transparency more than normal. It was stated that "having a member with excessive transparency attitude means more unnecessary distraction which may affect the workflow of the team progress". Moreover, lack of collaboration may happen as a result of 
Table 2. Examples of risks related to different levels of online social transparency

\begin{tabular}{|c|c|c|c|}
\hline & $\begin{array}{l}\text { Excessive level } \\
\text { of Transparency }\end{array}$ & $\begin{array}{c}\text { Normal level } \\
\text { of Transparency }\end{array}$ & $\begin{array}{c}\text { Lack } \\
\text { of Transparency }\end{array}$ \\
\hline Performance & $\begin{array}{l}\text { Employee Isolation } \\
\text { Lack of collaboration } \\
\text { Information overload } \\
\text { Slow Decision Making }\end{array}$ & $\begin{array}{l}\text { Loss of interest } \\
\text { low engagement } \\
\text { Low innovation } \\
\text { Social Loafing }\end{array}$ & $\begin{array}{l}\text { Conflict of interest } \\
\text { Loss of interest } \\
\text { Lack of collaboration }\end{array}$ \\
\hline Wellbeing & $\begin{array}{l}\text { Inadequate and unpre- } \\
\text { pared } \\
\text { Confusion in intentions } \\
\text { Stress } \\
\text { Pressure }\end{array}$ & $\begin{array}{l}\text { Stress \& Pressure } \\
\text { Low self-esteem } \\
\text { Negative impression } \\
\text { Distrust }\end{array}$ & $\begin{array}{l}\text { Lack of belonging } \\
\text { Relationship Conflict } \\
\text { Annoyance } \\
\text { Lack of trust }\end{array}$ \\
\hline $\begin{array}{c}\text { Workplace } \\
\text { Environment }\end{array}$ & $\begin{array}{l}\text { Uncomfortable Place } \\
\text { Loss of concentration } \\
\text { Loss of professionalism } \\
\text { Employees Turnover }\end{array}$ & $\begin{array}{l}\text { Favouritism } \\
\text { Disengagement } \\
\text { Discouraged employees }\end{array}$ & $\begin{array}{l}\text { Rumours spread } \\
\text { Biased opinions } \\
\text { Fabricated reactions } \\
\text { Information inaccuracy }\end{array}$ \\
\hline
\end{tabular}

creating information overload due to the excessive transparency of information related to a person's works. Information overload may also slow the decision-making process due to the quantity of information that needs to be processed.

Wellbeing. Excessive transparency may also lead to a risk of making employees feel inadequate or unprepared when they receive too much information, particularly irrelevant information about others' work. A participant stated that "I may receive information that I do not need to know but because it is sent to me, I feel like it is something I am expected to part of, or to understand". It was also showed that too much transparency might create confusion about the ultimate intention of this transparency which, therefore, creates a chance of making mistakes and waste time in the workplace. We noted that employees who are excessively transparent about their good performance run the risk of creating unwanted stress and pressure for employees who may constantly be thinking of how their performance impacts the team productivity.

Workplace environment. Social transparency in the workplace may make employees excessively open about their personal life more than the work which makes the workplace lose its professionalism. Distraction can stem from excessive transparency and make the workplace an unhealthy and uncomfortable environment for employees who may lose concentration to accomplish their work which in turn increase the rate of employees' turnover.

Risks related to normal online social transparency. In this section, we present the risks that may stem from a normal level of transparency. We identify this level as the required level of transparency when the shared information is seen by the audiences as satisfactory and beneficial to certain enterprise goals and activities. We noticed that even if transparency is seen adequate, it might lead to a negative impact on the rela- 
tionship between enterprise members and the level of trust and interest between them and may need further qualification and support with additional measures to mitigate risks on performance, wellbeing and the workplace environment.

Performance. ESS such as Slack typically have features that allow each member to pin certain messages to appear all the time to all team members as a headline or priority. However, using this feature to be transparent about certain facets such as the long duration or difficulty of a certain task may make colleagues lose their interest to contribute or collaborate in this task. We also found that employees tend to share their personal skills in solving certain problems either to promote their abilities or to make others learn from them. However, this kind of voluntary transparency, despite being relevant and needed, may reduce the innovation and creativity of other employees to find clever solutions and make them more reliant on those who have them. Besides the influence on individual performance, team performance may also be affected by social transparency. For example, our analysis showed that when employees are transparent about their interest in certain tasks; other members may be less motivated to work hard on behalf of the group. This loss of motivation is called social loafing and is associated with free riding. We noted that social transparency might trigger social loafing and free-riding in large groups where more than one member works in the same task or goal and traceability and auditing are harder.

Wellbeing. Employee personal, social and financial wellbeing at the workplace is a requirement for enterprises to address and ensure that their employees remain satisfied, motivated and loyal at work [16]. Wellbeing risks such as stress and pressure may stem from transparency when the information reveals conflict with other member's interest or goals. Transparency about work progress in the public channels in ESS, despite being needed for scheduling and coordination, might reach an employee who is less skilled or has less experience which consequently lowers their self-esteem and may also have a chance to leave a bad impression about others' progress if the information reached a high authority such as project manager. In addition, team collective wellbeing may also be affected by social transparency amongst the members. We noted that a team could suffer from a distrust problem due to misusing the information that is provided by other team members. For example, employees may share the reasons which slow their progress to eliminate high expectations or to seek voluntary help from other members. Less collaborative colleagues may use this information as a defence strategy and avoid blame on the progress of the whole team in the end. This is because the information in online social transparency are archived and can be retrieved later and processed to generate reports. This is similar to when people use the timeline or post history on social media to retrieve evidence of some social events and behaviours.

Workplace environment. We found that social transparency may lead to creating favouritism culture in the workplace. Favouritism is defined as special privileges or treatment provided to one person over all of the other employees [17]. Social transparency may develop friendship amongst employees who share similar interests, skills or experiences. This friendship may lead to creating special treatment for some employees over others. The favouritism that emerges from social transparency correlates with a feeling of disengagement from work, feeling discouraged by non-favoured 
employees. Common risks which were mentioned by our employee participants included nepotism amongst employees and the unjustifiable decisions made based on the special relationships with decision-makers.

Risks related to lack of online social transparency. Lack of transparency refers to the unintentional and occasional holding of individual's social information in the enterprise social software. We reiterate here that social transparency is not enforced by the organisational rules and left as a personal choice for staff. We noted that when there is no social transparency, it would be difficult for employees to know what is going on, why certain things are happening, and they may find themselves vulnerable, insecure and afraid of uncertainty. This typically leads to searching for precautionary and defensive strategies and following a more conservative and less creative attitude.

Performance. Lack of social transparency between team members or organisation members means communicating little or no information about colleagues' intentions towards their work, their interests in certain kinds of activities, their availabilities for future collaboration or justifications for unexpected actions. We found that when an employee fails to know about other's intentions such as their priorities and interests in certain tasks, that may create a conflict in performing these tasks and spend significant time in the least priority tasks. Loss of interest and lack of collaboration are other risks that result from lack of social transparency amongst enterprise members. For example, no transparency about interest in performing collaborative tasks may demotivate employees and make them think carefully before engaging in this task.

Wellbeing. We noted that a lack of belonging is one of the common issues that result from a lack of social transparency between peers in the same team. Lack of social transparency has been seen as a reason for relationship conflict because employees are unaware of other members' diverging interests and incompatible preferences which make employees misattribute the intentions of others. As a result, risks such as tension, annoyance, low work satisfaction and commitment, lack of trust and low group cohesion has a high chance to stem amongst employees.

Workplace environment. Our analysis showed that when social transparency is lacking amongst employees, team leaders and management members, there tends to be a high chance that rumours, biased opinions, inaccurate information and fabricated reactions will become common throughout the organisation processes and particularly employees' communication.

\subsection{Risks Related to Transparency Sharing Practice}

Research on social transparency describes it in a model where two parties exchange their information, and an observer has an opportunity to engage in these exchanges [13]. In the two companies, we observed the sharing practice amongst employees when conveying social transparency. Risks can be organised around two main types of social transparency practice: asymmetric and symmetric. A summary is presented in Table 3.

Risks related to symmetric online social transparency. This type is identified as the reciprocal transparency behaviour where the two parties are, at a certain point of 
time, transparent about their information and have enough information about each other. By observing symmetric social transparency in ESS, we concluded that the quality facets such as presentation, timing, and relevance of the information are the essential triggers of risks in the workplace.

Performance. Symmetric social transparency in ESS might create information overload to employees who are not interested in this information. In other words, people may not expect or want reciprocal transparency as a return to being transpar ent. We noted that unmanaged symmetric transparency could increase the distraction from work unless staff are enabled to filter responses to their transparency. Symmetrical social transparency and the perception of others to reciprocate transparency even if not demanded to do so can create a massive information history in the online platform, which may cost the employee time and effort to search for relevant information.

Wellbeing. Conditional reciprocity is an interesting noted behaviour in symmetric social transparency. Employees would be socially transparent when their colleagues are transparent as well. If the other party continually fails to be transparent, it will be reputational, and other employees will stop being transparent with them. That would add pressure on employees to avoid losing transparency of others as well as avoid Fear of Missing Out (FoMO) feelings occurring when they expect a return to their transparency from colleagues. FoMO is described in [18] as "the desire to be continually connected with what others are doing".

Workplace Environment. As mentioned earlier, some employees may not expect or like reciprocal transparency, i.e. transparency from others as a return to their own transparency. A workplace with symmetrical transparency behaviour might be an uncomfortable place for them as it can create a less genuine sharing practice and workarounds. For example, we noted that symmetrical transparency behaviour does not ensure that information itself is also symmetrical in quality, timeliness and format which can increase the chance for reducing harmony and consistency in the workplace. We noted that employees who practice symmetrical transparency might lose the opportunity to learn from their colleagues. For example, when expert employees feel they only need to be transparent about their progress and tasks and hold the information related to the techniques they use knowing that novice colleagues would not be able to reciprocate in that aspect. Symmetrical transparency is also described by some of our participants as a sign of lack of trust in the workplace as employees may not feel secure to be fully and truly transparent but rather pressured to do that.

Risks related to asymmetric online social transparency. This type occurs when one party is more transparent, in terms of information content and, also timing and proactiveness than other parties. Asymmetric transparency can make a discrepancy in situational awareness. As social transparency is voluntary, there are no regulations to oblige employees to be transparent with each other and how to choose the time and frequency for doing so. 
Table 3. Examples of risks related to Symmetric and Asymmetric Online Social Transparency

\begin{tabular}{|c|c|c|}
\hline & $\begin{array}{c}\text { Symmetric Social } \\
\text { Transparency }\end{array}$ & $\begin{array}{c}\text { Asymmetric Social } \\
\text { Transparency }\end{array}$ \\
\hline Performance & $\begin{array}{l}\text { Information overload } \\
\text { Distraction } \\
\text { Big information history } \\
\text { Time/effort consuming }\end{array}$ & $\begin{array}{l}\text { Insufficient knowledge } \\
\text { Delay in progress } \\
\text { Low performance and productivity }\end{array}$ \\
\hline Wellbeing & $\begin{array}{l}\text { Conditional reciprocity } \\
\text { Pressure } \\
\text { FoMO }\end{array}$ & $\begin{array}{l}\text { Power imbalance } \\
\text { Stress } \\
\text { Insecure employees } \\
\text { Pressure }\end{array}$ \\
\hline $\begin{array}{c}\text { Workplace } \\
\text { Environment }\end{array}$ & $\begin{array}{l}\text { Uncomfortable place } \\
\text { Loss learning opportunity } \\
\text { Low harmony }\end{array}$ & $\begin{array}{l}\text { Low group cohesion } \\
\text { Insecure workplace } \\
\text { Unfair workplace }\end{array}$ \\
\hline
\end{tabular}

Performance. We noted that there is a chance to reduce collaboration amongst employees who are not transparent or less transparent about their information at the time others are transparent. Risks such as insufficient knowledge base due to inequivalent communication between members of an organisation and low consistency of transparency behaviour play a significant role in delaying the employee's progress, lowering their performance and reducing the overall productivity of the organisation.

Wellbeing. Asymmetric social transparency can create power imbalance as individuals may use others' information as a way to empower themselves or misuse the information for personal benefits such as complaining against an employee to relocate them to a different department. With the characteristics of digital systems, such as data retrieval, real-time and traceability, e.g. sharing a location, these risks are maximised if we compare them to face-to-face transparency. From a collaboration perspective, there is a high chance to reduce collaboration with employees who are not transparent or less transparent about their information compared with their colleagues. Employees may have stress and insecure feelings to collaborate or engage in a work with an employee that is less transparent than them. We found that the asymmetric transparency behaviour adds pressure to employees to cope with the behaviour of more transparent colleagues. A participant declares that this pressure may happen for employees who tend to cope with other behaviour to create a good impression or to avoid any blame for less transparent behaviour.

Workplace environment. Asymmetric behaviour of social transparency has a chance to reduce group cohesion due to the imbalanced transparency amongst employees. A participant described a workplace with asymmetric transparency as an insecure workplace. It has also been described as an unfair workplace because_this 
behaviour decreases the learning opportunity amongst employees, particularly new or less-skilled employees.

\section{Discussion}

Work on organisational transparency showed the positive impact of social transparency in motivating peer production and accelerating the decision-making process [19]. As discussed in this paper, social transparency in ESS can enable enterprise members to adjust their inter-relationships with others, expectations, focus, and priorities and be aware of the dynamic contexts of their group and enterprise. The ultimate goal of social transparency is to enable the enterprise to reach its strategic goals and at the same time maintain quality and social requirements such as job satisfaction and perception of openness and fairness [3]. However, in the absence of shepherding and guidance on how individuals practice social transparency via the ESS, especially about the level of transparency (in amount, subject, outreach, and frequency) and the balance of sharing and openness culture amongst all, the intended benefits of it can be easily compromised.

Although the literature in fields like Computer Supported Cooperative Work (CSCW) conceptualised social transparency as information sharing technique, mainly practices within small group settings, we still lack methods to engineer such online platforms to make decisions about social transparency informed, especially across a large scale such as enterprise. For example, an individual would need the system to predict and visualise the impact of sharing certain information and, also, receive impressions and feedback on the sharing, which help them refine future actions. We argued in [3] that EES also needs to provide a more structured way which allows better management of the content of transparency, interaction time and the set of audience, still without contradicting with the free-spirit in social transparency and its voluntary nature and reliance on an openness culture.

Besides the need for automated support to assist individuals in making an informed decision about social transparency, assessing its risks can be integrated into the design phase of social enterprise software, so that common risks are elicited and dealt with in advance as part of the design and its interactive features. For example, capturing the strategic goals and activities of each role in the organisation can become a reference point for decisions around the relevance of information content and hence be a basis for decisions around information overload. We suggest that such an assessment method has to include two phases: the preparation phase and the analysis and actioning phase. We intend to consolidate and validate our proposed phases in future work.

Preparation phase: This phase is expected to be administered by the system analysts alongside enterprise management, and it shall also include representatives of each role in the enterprise. This phase is to set up the scene and to determine the parties involved in the assessment and loci in the business process where social transparency can be beneficial or detrimental. The decision about transparency risks are subjective and differ from one individual to another and even for the same individual depending on their context. Early in this paper, we defined social transparency as a voluntary act to share information about the individual's own status including goals, activities, priorities, mood, plans, and skills. Therefore, and given the nature of infor- 
mation and the personal differences in risk assessment, we suggest using humancentred techniques which allow capturing such diversity [20]. Examples include the use of user stories, goal modelling, think-aloud and scenarios to both generate test cases and speculated risks. We would need a bespoke version of such techniques to fit the peculiarities and special nature of social transparency, e.g. a domain-specific template for user stories supported with a controlled vocabulary reflecting typical intentions and goals of social transparency. In our previous work [3], we demonstrated that social transparency side-effect becomes evident once it is practised in the day to day life of the enterprise members. Hence, we suggest gathering user stories over some time and merging it with methods like diary studies [21]. User stories collected in real-world context and aided by a simulator can then be used to generate scenarios which will allow the system analysts and management to get real-life examples of the interaction, activities, and behaviour amongst enterprise members and their practice of social transparency in a realistic manner. To document and formalise social transparency and its risks within their organisational context, techniques like goal modelling and BPMN can be used so that formal analysis can be then conducted.

Analysis and Actioning phase: The user-centred techniques (user stories, diaries, scenarios and think aloud) and the presentation of the knowledge captured through them (goal modelling and BPMN) built in the preparation phase are meant to be the knowledge base for the analysis and actioning phase. Approached involving the actual users of EES and social transparency, such as participatory design approach [22], are preferred given the nature of risks, i.e., being subjective and context-dependent. In [3] we advocated mitigation strategies can lead to further risks. For example, mitigating risks of lack of cooperation due to asymmetric transparency through increasing openness may lead to triggering information overload risk. Therefore, the method would need to look at the chain of risks and their impact and weight, so it allows prioritisation. Moreover, social transparency is not only an individual act, but it also involves all members of the enterprise. Therefore, we suggest that the assessment process has to be performed collectively so that it captures emerging properties and become more sensitive to group dynamics. As such risks can emerge only in a real-world context, despite making all effort to predict them at the design stage, iterative and lifelong risk assessment methods within the enterprise information systems would be needed.

\section{Conclusion}

In our previous works, we provided the basics of assessing social transparency and its associated risks related to the delivery of information which includes the content, presentation, timeliness and transparency recipients. This paper is complementary to our previous work on assessing online social transparency in the enterprise. We provided categories of online social transparency based on the awareness of transparency provider and the level of accessibility to the information. In terms of the risks of social transparency, we have considered here the influence of the level of transparency and the transparency sharing practice on performance, wellbeing and workplace environment. Our future works will design an engineering method that aid system analysts and enterprise management to assess social transparency implemented in their online platforms. 


\section{References}

1. Graupner, S., et al. When social media meet the enterprise. In IEEE 16th International Enterprise Distributed Object Computing Conference (EDOC). pp. 201-210, IEEE, (2012).

2. Pavlíček, A. and P. Doucek. Social Media and Social CRM. In International Conference on Research and Practical Issues of Enterprise Information Systems (CONFENIS). 2016, pp. 77-87, Springer, (2016).

3. Alsaedi, T., K. Phalp, and R. Ali, Towards an Assessment Method for Social Transparency in Enterprise Information Systems. In the $10^{\text {th }}$ Workshop on Service oriented Enterprise Architecture for Enterprise Engineering (SoEA4EE'19), colocated with the $23^{\text {rd }}$ IEEE international Enterprise Computing Conference (EDOC), IEEE, (2019).

4. Pavlíček, A. and P. Doucek. Corporate social responsibility in social media environment. In Information and Communication Technology-EurAsia Conference (CONFENIS), pp.323-332, Springer, (2015).

5. Gibbons, R., et al., What do managers do? Suggestive evidence and potential theories about building relationships. Handbook of Organizational Economics, Princeton University Press, Princeton, NJ, forthcoming, (2010).

6. Brandes, L. and D. Darai, The value and motivating mechanism of transparency in organizations. European Economic Review 98, 189-198 (2017).

7. Schnackenberg, A.K. and E.C. Tomlinson, Organizational transparency: A new perspective on managing trust in organization-stakeholder relationships. Journal of Management, 42(7), 1784-1810 (2016).

8. Laud, R.L. and D.H. Schepers, Beyond transparency: Information overload and a model for intelligibility. Business and Society Review, 114(3), 365-391 (2009).

9. Parris, D.L., et al., Exploring transparency: a new framework for responsible business management. Management Decision, 54(1), 222-247 (2016).

10. do Prado Leite, J.C.S. and C. Cappelli. Exploring i* Characteristics that Support Software Transparency. In iStar, (2008).

11. Hosseini, M., et al. A modelling language for transparency requirements in business information systems. In International Conference on Advanced Information Systems Engineering (CAiSE), LNCS, vol.9694, pp.239-254, Springer, Cham (2016).

12. Dabbish, L., et al. Social coding in GitHub: transparency and collaboration in an open software repository. In Proceedings of the conference on Computer Supported Cooperative Work (CSCW). pp.1277-1286, ACM, (2012).

13. Stuart, H.C., et al. Social transparency in networked information exchange: a theoretical framework. In Proceedings of the conference on Computer Supported Cooperative Work (CSCW), pp.451-460, ACM, (2012).

14. Alsaedi, T., Phalp, K., Ali, R., Social Transparency in Enterprise Information Systems: Peculiarities and Assessment Factors. In The 6th International Conference on Behavioral, Economic, and Socio-Cultural Computing (BESC), IEEE, 2019.

15. Braun, V. and V. Clarke, Using thematic analysis in psychology. Qualitative research in psychology, 3(2): p. 77-101, (2006). 
16. Renee Baptiste, N., Tightening the link between employee wellbeing at work and performance: A new dimension for HRM. Management decision, 46(2), 284309(2008).

17. Arasli, H., H.E. Arici, and N. Çakmakoğlu Arici, Workplace favouritism, psychological contract violation and turnover intention: Moderating roles of authentic leadership and job insecurity climate. German Journal of Human Resource Management,33(3), 197-222 (2019).

18. Przybylski, A.K., et al., Motivational, emotional, and behavioral correlates of fear of missing out. 29(4), 1841-1848 (2013).

19. Marlow, J. and L.A. Dabbish. The effects of visualizing activity history on attitudes and behaviors in a peer production context. In Proceedings of the 18th ACM Conference on Computer Supported Cooperative Work \& Social Computing (CSCW), pp.757-764, ACM, (2015).

20. Giacomin, J., What is human centred design? The Design Journal, 17(4), 606-623 (2014).

21. Singer, J., S.E. Sim, and T.C. Lethbridge, Software engineering data collection for field studies, In Guide to Advanced Empirical Software Engineering, Springer. p. 9-34, (2008).

22. Spinuzzi, C., The methodology of participatory design. Technical communication, 52(2), 163-174, (2005). 